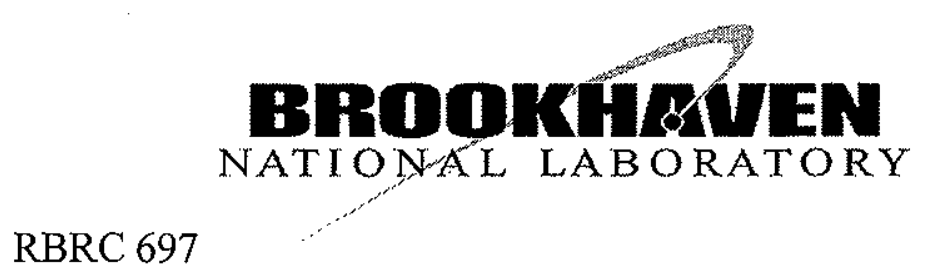

BNL-79726-2007-CP

\title{
Phase structure of twisted Eguchi-Kawai model
}

\author{
Ishikawa, T.; Azeyanagi (Kyoto U.), T.; Hanada, M. (RIKEN); \\ Harata, T. (Kyoto U.)
}

The XXV International Symposium on Lattice Field Theory

Regensburg, Germany

July 30 - August 4, 2007

\author{
Physics Department/RIKEN BNL Research Center \\ Brookhaven National Laboratory \\ P.O. Box 5000 \\ Upton, NY 11973-5000 \\ www.bnl.gov
}

Notice: This manuscript has been authored by employees of Brookhaven Science Associates, LLC under Contract No. DE-AC02-98CH10886 with the U.S. Department of Energy. The publisher by accepting the manuscript for publication acknowledges that the United States Government retains a non-exclusive, paid-up, irrevocable, world-wide license to publish or reproduce the published form of this manuscript, or allow others to do so, for United States Government purposes. 


\section{DISCLAIMER}

This report was prepared as an account of work sponsored by an agency of the United States Government. Neither the United States Government nor any agency thereof, nor any of their employees, nor any of their contractors, subcontractors, or their employees, makes any warranty, express or implied, or assumes any legal liability or responsibility for the accuracy, completeness, or any third party's use or the results of such use of any information, apparatus, product, or process disclosed, or represents that its use would not infringe privately owned rights. Reference herein to any specific commercial product, process, or service by trade name, trademark, manufacturer, or otherwise, does not necessarily constitute or imply its endorsement, recommendation, or favoring by the United States Government or any agency thereof or its contractors or subcontractors. The views and opinions of authors expressed herein do not necessarily state or reflect those of the United States Government or any agency thereof. 


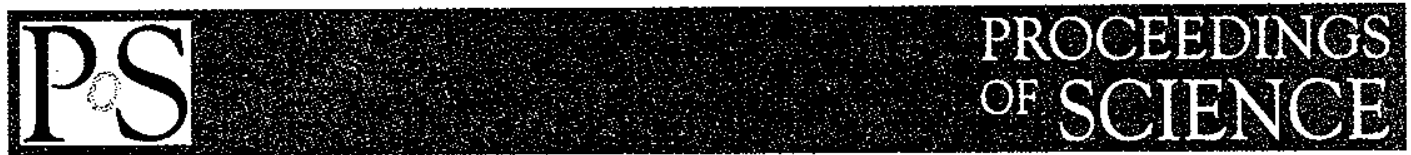

\title{
Phase structure of twisted Eguchi-Kawai model
}

$\sqrt{\text { Tomomi Ishikawa* }}$

RIKEN BNL Research Center, Brookhaven National Laboratory, Upton, New York 11973, USA

E-mail: tomomilequark.phy.bnl.gov

Tatsuo Azeyanagi

Department of Physics, Kyoto University, Kyoto 606-8502, Japan

\section{Masanori Hanada}

Theoretical Physics Laboratory, RIKEN Nishina Center, Wako, Saitama 351-0198, Japan

\section{Tomoyoshi Hirata}

Department of Physics, Kyoto University, Kyoto 606-8502, Japan

\begin{abstract}
We study the phase structure of the four-dimensional twisted Eguchi-Kawai model using numerical simulations. This model is an effective tool for studying $S U(N)$ gauge theory in the large- $N$ limit and provides a nonperturbative formulation of the gauge theory on noncommutative spaces. Recently it was found that its $\mathbb{Z}_{N}^{4}$ symmetry, which is crucial for the validity of this model, can break spontaneously in the intermediate coupling region. We investigate in detail the symmetry breaking point from the weak coupling side. Our simulation results show that the continuum limit of this model cannot be taken.
\end{abstract}

The XXV International Symposium on Lattice Field Theory

July 30 - August 42007

Regensburg, Germany

${ }^{*}$ Speaker. 


\section{Introduction}

In 1982, Eguchi and Kawai introduced an important and interesting idea, which is now called Eguchi-Kawai equivalence [1]. Consider the $S U(N)$ gauge theory (YM) on a periodic $D$-dimensional lattice with the Wilson plaquette action. In the large- $N$ limit the space-time degrees of freedom can be neglected, and the theory is then equivalent to a model defined on a single hyper-cube, called the Eguchi-Kawai model (EK model). This correspondence was shown by observing that the Schwinger-Dyson equations for Wilson loops (loop equations) in both theories are the same. Naìvely, in the EK model the loop equations can have open Wilson lines, which do not exist in the original gauge theory due to gauge invariance. Therefore we need to assume that the global $\mathbb{Z}_{N}^{D}$ symmetry $U_{\mu} \rightarrow e^{i \theta_{\mu}} U_{\mu}$, which prohibits non-zero expectation values of the open Wilson lines, is not broken spontaneously. However, soon after the discovery of the equivalence, it was found that the symmetry is actually broken for $D>2$ in the weak coupling region [2]. Although the naive EK equivalence does not hold, several modifications have been proposed for this issue; they are the "quenched" Eguchi-Kawai model (QEK model) [2, 3, 4] and the "twisted" Eguchi-Kawai model (TEK model) [5]. Historically, more work has been performed on the TEK model because it is theoretically interesting and numerically more practical. In addition, this model also describes gauge theories on noncommutative spaces (NCYM) [6,7].

The TEK model is a matrix model defined by the partition function

$$
Z_{T E K}=\int \prod_{\mu=1}^{D} d U_{\mu} \exp \left(-S_{T E K}\right)
$$

with the action

$$
S_{T E K}=-\beta N \sum_{\mu \neq v} Z_{\mu v} \operatorname{Tr} U_{\mu} U_{v} U_{\mu}^{\dagger} U_{v}^{\dagger}, \quad \beta=1 / g^{2},
$$

where $d U_{\mu}$ and $U_{\mu}(\mu=1, . ., D)$ are Haar measure and link variables. The phase factors $Z_{\mu \nu}$ are

$$
Z_{\mu v}=\exp \left(2 \pi i n_{\mu v} / N\right), \quad n_{\mu v}=-n_{\nu \mu} \in \mathbb{Z}_{N} .
$$

The classical solution $U_{\mu}^{(0)}=\Gamma_{\mu}$ satisfies the 't Hooft algebra

$$
\Gamma_{\mu} \Gamma_{v}=Z_{v \mu} \Gamma_{v} \Gamma_{\mu},
$$

and is called "twist-eater". The twist-eater guarantees existence of the $\mathbb{Z}_{N}^{D}$ symmetry in the weak coupling limit. It is unclear whether or not the symmetry is unbroken in the intermediate coupling region, as there is no guarantee the symmetry is preserved. Numerical simulations performed during the early days of this model, however, showed that the symmetry is unbroken throughout the whole coupling region. This has encouraged the belief that the TEK model describes the large$N$ limit of $S U(N)$ gauge theory.

Surprisingly, some indications of $\mathbb{Z}_{N}^{D}$ symmetry breaking were recently reported in several contexts concerning the TEK model $[8,9,10]$. In $[10]$, the $D=4$ TEK model with standard twist was investigated up to $N=144$ and $\mathbb{Z}_{N}^{4}$ symmetry breaking phenomena in the intermediate coupling region was observed by Monte-Carlo simulations. The work presented in this article continues this investigation. We concentrate on investigating the locations of the symmetry breaking from the weak coupling side in the $(\beta, N)$ plane to determine if the continuum limits of the TEK models can be taken as the YM and the NCYM. 


\section{Twist prescriptions}

In this study we treat the $D=4$ case. Among the various types of twist possible, we apply:

$$
\begin{array}{llll}
n_{\mu \nu}=L \varepsilon_{\mu \nu}^{\text {sym }}, & N=L^{2} & & \text { (minimal symmetric twist, standard twist), } \\
n_{\mu \nu}=L \varepsilon_{\mu \nu}^{\text {skew }}, & N=L^{2} & & \text { (minimal skew-diagonal twist) } \\
n_{\mu \nu}=m L \varepsilon_{\mu \nu}^{\text {skew }}, & N=m L^{2} & & \text { (generic skew-diagonal twist) }
\end{array}
$$

where we define anti-symmetrization matrices as

$$
\varepsilon_{\mu \nu}^{\text {sym }}=\left(\begin{array}{cccc}
0 & 1 & 1 & 1 \\
-1 & 0 & 1 & 1 \\
-1 & -1 & 0 & 1 \\
-1 & -1 & -1 & 0
\end{array}\right), \quad \varepsilon_{\mu \nu}^{\text {skew }}=\left(\begin{array}{cc|cc}
0 & 1 & 0 & 0 \\
-1 & 0 & 0 & 0 \\
\hline 0 & 0 & 0 & 1 \\
0 & 0 & -1 & 0
\end{array}\right)
$$

These twists represent $L^{4}$ lattices. The symmetric and the skew-diagonal form can be transformed into one another by an $S L(4, \mathbb{Z})$ transformation for the coordinates on $\mathbb{T}^{4}$ [11]. While these forms differ only by a coordinate transformation, they can give different results except the weak coupling limit. We note that the generic twist (2.3) can be regarded as the gauge theory on $m$-coincident fuzzy $\mathbb{T}^{4}$. (The minimal twists $(2.1)$ and (2.2) are particular cases $(m=1)$ of the generic twist.)

\section{Theoretical discussion for the $\mathbb{Z}_{N}^{4}$ symmetry breaking in the TEK model}

As we mentioned in the introduction, the $\mathbb{Z}_{N}^{4}$ symmetry can be broken in the intermediate coupling region. In this section we give a theoretical discussion about this phenomena.

Here, we consider the first breaking point from the weak coupling limit $\beta_{c}^{L}$. We assume that $\mathbb{Z}_{N}^{4}$ symmetry breaking at this point is a transition from the twist-eater phase $U_{\mu}=\Gamma_{\mu}$ to the identity configuration phase $U_{\mu}=\mathbb{1}_{N}{ }^{1}$ For simplicity we consider a $\mathbb{Z}_{N}^{4} \stackrel{\beta_{c}^{L}}{\longrightarrow} \mathbb{Z}_{N}^{0}$ type transition here. Of course we can treat $\mathbb{Z}_{N}^{4} \stackrel{\beta_{c}^{L}}{\longrightarrow} \mathbb{Z}_{N}^{3} \stackrel{\beta_{c}^{L}}{\longrightarrow} \mathbb{Z}_{N}^{2} \stackrel{\beta_{c}^{L}}{\longrightarrow} \mathbb{Z}_{N}^{1} \stackrel{\beta_{c}^{L}}{\longrightarrow} \mathbb{Z}_{N}^{0}$ (cascade) type breaking, but the obtained behavior is not different from the former type. First, we focus on the classical energy difference between these configurations. The energy difference can be easily calculated from the action (1.2) as

$$
\begin{aligned}
\Delta S & =S_{T E K}\left[U_{\mu}=\mathbb{1}_{N}\right]-S_{T E K}\left[U_{\mu}=\Gamma_{\mu}\right] \\
& =\beta N^{2} \sum_{\mu \neq v}\left\{1-\cos \left(\frac{2 \pi n_{\mu \nu}}{N}\right)\right\} \simeq 2 \pi^{2} \beta \sum_{\mu \neq v} n_{\mu \nu}^{2} .
\end{aligned}
$$

For the generic twist we have

$$
\Delta S= \begin{cases}24 \pi^{2} \beta m^{2} L^{2} & \text { (symmetric form) } \\ 8 \pi^{2} \beta m^{2} L^{2} & \text { (skew-diagonal form) }\end{cases}
$$

\footnotetext{
${ }^{1}$ Of course, the twist-eater only has $\mathbb{Z}_{L}^{4}$ symmetry for the twists we apply. But we write it as $\mathbb{Z}_{N}^{4}$ in this article.
} 
Note that the symmetric form is roughly three times more stable than the skew-diagonal form if both twists have similar quantum fluctuations. Thus the $\mathbb{Z}_{N}^{4}$ symmetry breaking for the skew-diagonal form can occur at smaller $N$ than that for the symmetric form.

Going away from the weak coupling limit, the system experiences greater quantum fluctuations. Here, we naively expect that the $\mathbb{Z}_{N}^{4}$ symmetry is broken if the fluctuation around the twist-eater configuration exceeds the energy difference $\Delta S$. Because the system describes $O\left(N^{2}\right)$ interacting gluons, it is natural to assume that their quantum fluctuations provide an $O\left(N^{2}\right)$ contribution to the effective action. For the generic twist, the fluctuation is $O\left(m^{2} L^{4}\right)$. Combining this with eq. (3.2), we can estimate the critical point $\beta_{c}^{L}$ as

$$
\beta_{c}^{L} \sim L^{2}
$$

Although the above discussion is quite crude, the symmetry breaking behavior described by (3.3) is consistent with the numerical results discussed in the next section.

\section{Numerical simulations}

In this section we show the results of our numerical simulations for the $\mathbb{Z}_{N}^{4}$ symmetry breaking phenomena. In order to discuss the continuum and large- $N$ limits for this model, we concentrate on the first breaking point from the weak coupling side.

\subsection{Simulation method}

In our simulation we use the pseudo-heatbath algorithm. The algorithm is based on [12], and in each sweep over-relaxation is performed five times after multiplying $S U(2)$ subgroup matrices. The number of sweeps is $O(1000)$ for each $\beta$. We scanned for the symmetry breaking point with a resolution of $\Delta \beta=0.005$, and thus quote \pm 0.0025 as the error due to the finite resolution. Note that the breaking points are ambiguous because the breakdown of the $\mathbb{Z}_{N}^{4}$ symmetry is a firstorder transition. As an order parameter for detecting the symmetry breakdown, we measure the expectation value of Polyakov lines

$$
P_{\mu} \equiv\left|\left\langle\frac{1}{N} \operatorname{Tr} U_{\mu}\right\rangle\right| .
$$

\subsection{Simulation results}

First we consider the minimal twists. Figures 1 and 2 show the critical lattice coupling from the weak coupling side $\beta_{c}^{L}$ for the symmetric and skew-diagonal twists, respectively. For the minimal skew-diagonal twist we also observe the critical lattice coupling from strong coupling side $\beta_{c}^{H}$. We see that while the $\mathbb{Z}_{N}^{4}$ symmetry begins to break at $N=100$ for the symmetric form, it is already violated at $N=25$ for the skew-diagonal form, which is consistent with the theoretical considerations in section 3. Additionally, we observe a clear linear dependence of $\beta_{c}^{L}$ on $N\left(=L^{2}\right)$ :

$$
\begin{aligned}
& \beta_{c}^{L} \sim 0.0011 N+0.21 \text {. (minimal symmetric twist) } \\
& \beta_{c}^{L} \sim 0.0034 N+0.25 \quad \text { (minimal skew-diagonal twist), }
\end{aligned}
$$




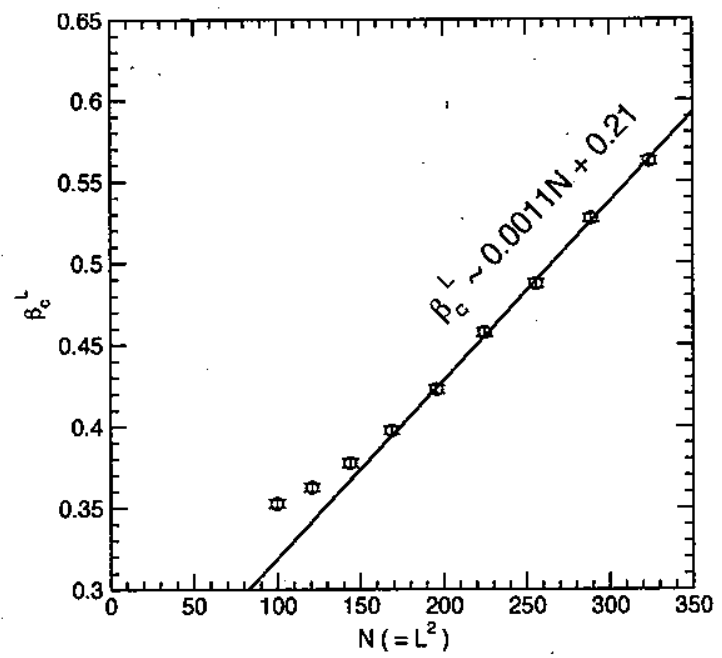

Figure 1: $\beta_{c}^{L}$ versus $N$ (minimal symmetric twist). Fit line is eq. (4.2), which is obtained using $N \geq$ 169 data.

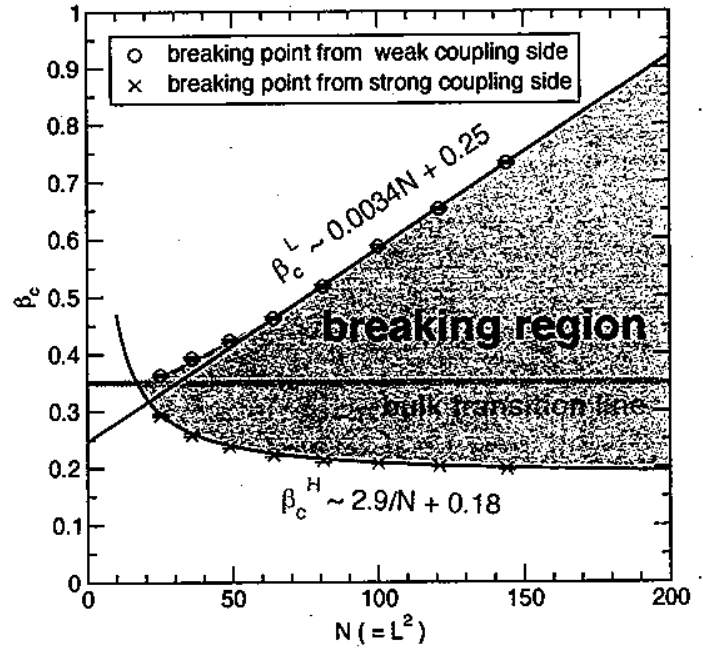

Figure 2: $\beta_{c}^{L}$ and $\beta_{c}^{H}$ versus $N$ (minimal skewdiagonal twist). Fit lines are eqs. (4.3) and (4.4), which are obtained using $N \geq 64$ and $N \geq 25$ data, respectively. The $\mathbb{Z}_{N}^{4}$ symmetry is broken within the light blue shaded area.

in the larger $N$ region. This behavior was already obtained in the theoretical discussion. Note that the coefficient of $N$ for the skew-diagonal twist is roughly three times larger than that for symmetric twist, which is also consistent with the theoretical analysis. For $\beta_{c}^{H}$, we find the relation

$$
\beta_{c}^{H} \sim 2.9 / N+0.18 \quad \text { (minimal skew-diagonal twist). }
$$

As $N$ is increased, $\beta_{c}^{H}$ approaches a point where the phase transition $\mathbb{Z}_{N}^{4} \stackrel{\beta_{c}^{H}}{\longrightarrow} \mathbb{Z}_{N}^{3}$ takes place in the original EK model.

For the generic twist we use the skew-diagonal form because $\mathbb{Z}_{N}^{4}$ symmetry breaking occurs at smaller $N$ than for the symmetric form, which makes our investigation much easier. We measure $\beta_{c}^{L}$ for this twist up to $m=4$. The simulation results are plotted in figure 3 . From this figure we find that for each $L$, the $\beta_{c}^{L}$ show weak $1 / m$ behavior. The points at $1 / m=0$ are linearly extrapolated values. ( $m=\infty$ means that an infinite number of fuzzy tori are superimposed.) The behavior for $L=5$ is particularly interesting. While $\mathbb{Z}_{N}^{4}$ symmetry breaking is observed for $m=1,2$, and 3 , it is not seen for $m=4$ because $\beta_{c}^{L}$ reaches a bulk transition point as $m$ is increased. Figure 4 represents the same data as figure 3 , but with $L^{2}$ as the horizontal axis. As we have seen in the minimal case, the data for $L \geq 8$ can be fitted by a linear function in $L^{2}$ for each $m$. From these figures, we discover that the data for $L \geq 8$ are well fitted globally by:

$$
\beta_{c}^{L} \sim 0.0034 L^{2}+\frac{0.060}{m}+0.19 \quad \text { (generic skew-diagonal twist) }
$$

The discussion in section 3 did not predict the observed dependence of $\beta_{c}^{L}$ on $1 / m$. While we do not have a clear reason for this phenomenon at present, we suspect that it is related to collective modes of the eigenvalues of the link variables. 


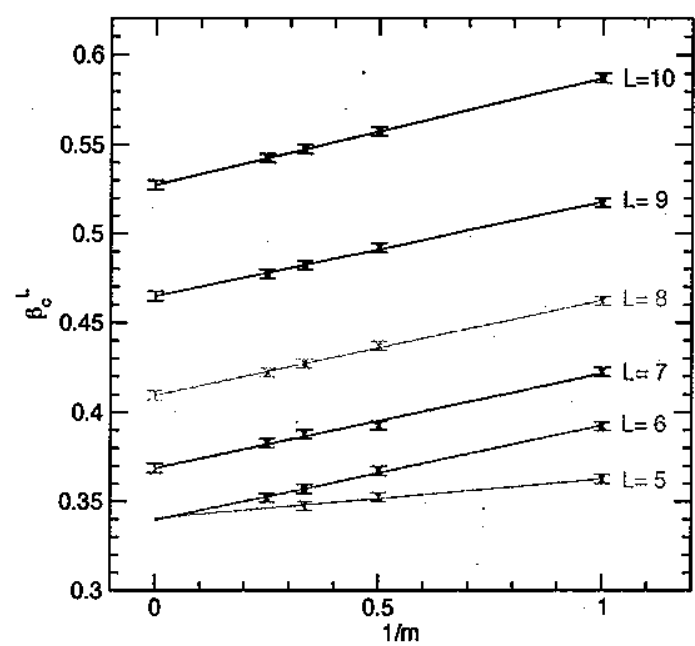

Figure 3: $\beta_{c}^{L}$ versus $1 / m$ for each $L$ (generic skewdiagonal twist). $\beta_{c}^{L}$ for $m=\infty$ is obtained by linear extrapolation.

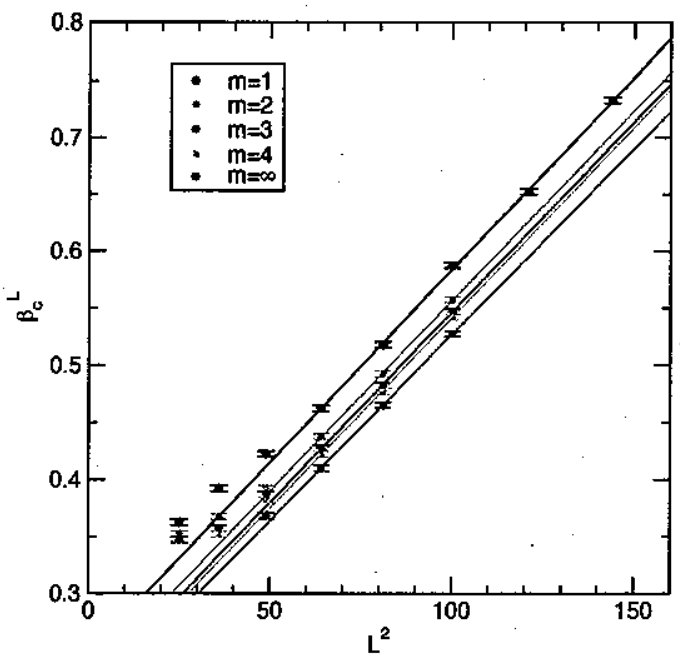

Figure 4: $\beta_{c}^{L}$ versus $L^{2}$ for each $m$ (generic skewdiagonal twist). We also include $m=\infty$ data, which is obtained by the extrapolation shown in figure 3

\section{Continuum limit}

Although our simulation is restricted to the small $N$ region, we may use our theoretical considerations to make statements about the large- $N$ limit. Thus the EK equivalence is valid only in the region $\beta>\beta_{c}^{L} \sim N$, not only in the smaller $N$ region, but also in the large- $N$ limit.

As is well known, the one-loop perturbative calculation of the YM lattice theory shows that its beta function behaves as $\beta \sim \log a^{-1}$ near the weak coupling limit, where $a$ is lattice spacing. If we wish to make the TEK model correspond to the YM theory, the scaling of the TEK model should obey that of the YM. In the TEK model, the lattice size $L$ is related to $N$. (For the generic twist, the relation is $N=m L^{2}$.) Then the continuum limit of the YM system with fixed physical size $l=a L$ can be obtained using the scaling

$$
\beta \sim \log a^{-1} \sim \log N
$$

In order to obtain the large- $N$ limit with infinite volume, we should keep $\beta$ lower than eq. (5.1). Otherwise, the system would shrink to a point. However, the simulation results obtained in this study show that $\beta_{c}^{L}$ grows faster than the logarithm. Therefore we conclude that EK equivalence breaks down and the TEK model does not have YM as its continuum limit.

In the case of the NCYM, the beta function is essentially the same as that of the YM theory at the one-loop level [13], and thus the scaling near the weak coupling limit is $\beta \sim \log a^{-1}$. But if we wish to make the TEK model correspond to the NCYM, there is the constraint $a^{2} L=$ fixed, which means that we take a scheme in which the noncommutative parameter $\theta$ is fixed. And then both the continuum limit and the infinite volume limit are simultaneously taken (double scaling limit). Regardless of the constraint, by the nature of the logarithm scaling, the scaling for the NCYM is the same as that of the ordinary YM (eq. 5.1). Therefore we conclude also that the TEK model does not have NCYM as its continuum limit. 


\section{Conclusions}

We carefully investigated the $\mathbb{Z}_{N}^{4}$ symmetry breaking phenomena in the TEK model using Monte-Carlo simulation. We found a clear linear dependence on $L^{2}$ for the symmetry breaking point from the weak coupling side. Regrettably, this means that the TEK models which use simple twists cannot be made to correspond to either ordinary YM or NCYM in the continuum limit.

Finally, we mention the partial reduction [14], which has been actively used in recent years. [14] showed that the large- $N$ reduction is valid above some critical physical size $l_{c}$. This means that for a lattice size $L$ the reduction holds only below some lattice coupling $\beta(L)$. In order to take continuum limit we should avoid the bulk transition at $\beta_{c}^{B}$, and thus there is a lower limit to the lattice size $L_{c}(\beta)$ used for the reduction. It is clever that the twist prescription is applied to this reduction [15], and we believe it would be very efficient.

\section{Acknowledgments}

The numerical computations for this work were carried out in part at the Yukawa Institute Computer Facility. M. H. is supported by Special Postdoctoral Researchers Program at RIKEN. T. H. would like to thank the Japan Society for the Promotion of Science for financial support.

\section{References}

[1] T. Eguchi and H. Kawai, Phys. Rev. Lett. 48 (1982) 1063.

[2] G. Bhanot, U. M. Heller and H. Neuberger, Phys. Lett. B 113 (1982) 47.

[3] G. Parisi, Phys. Lett. B 112 (1982) 463.

[4] D. J. Gross and Y. Kitazawa, Nucl. Phys. B 206 (1982) 440.

[5] A. Gonzalez-Arroyo and M. Okawa, Phys. Lett. B 120 (1983) 174;

A. Gonzalez-Arroyo and M. Okawa, Phys. Rev. D 27 (1983) 2397.

[6] H. Aoki, N. Ishibashi, S. Iso, H. Kawai, Y. Kitazawa and T. Tada, Nucl. Phys. B 565 (2000) 176.

[7] J. Ambjorn, Y. M. Makeenko, J. Nishimura and R. J. Szabo, JHEP 9911 (1999) 029;

J. Ambjorn, Y. M. Makeenko, J. Nishimura and R. J. Szabo, Phys. Lett. B 480 (2000) 399;

J. Ambjorn, Y. M. Makeenko, J. Nishimura and R. J. Szabo, JHEP 0005 (2000) 023.

[8] T. Ishikawa and M. Okawa, talk given by T. Ishikawa at the annual meeting of Japan Physics Society, Sendai, Japan, March 2003.

[9] W. Bietenholz, J. Nishimura, Y. Susaki and J. Volkholz, JHEP 0610 (2006) 042.

[10] M. Teper and H. Vairinhos, Phys. Lett. B 652 (2007) 359.

[11] P. van Baal and B. van Geemen, J. Math. Phys. 27 (1986) 455.

[12] K. Fabricius and O. Haan, Phys. Lett. B 143 (1984) 459.

[13] S. Minwalla, M. Van Raamsdonk and N. Seiberg, JHEP 0002 (2000) 020.

[14] R. Narayanan and H. Neuberger, Phys. Rev. Lett. 91 (2003) 081601.

[15] A. Gonzalez-Arroyo, R. Narayanan and H. Neuberger, Phys. Lett. B 631 (2005) 133. 